\title{
Investigación cualitativa de segundo orden y la comprensión de la realidad"
}

\author{
Julie Paola Lizcano Roa**
}

Recibido: 4 de febrero de 2012 Evaluado: 10 de marzo de 2012 Aceptado: 19 de abril de 2012

\section{Resumen}

El presente artículo resume algunas reflexiones teóricas y personales en torno a la importancia de la investigación cualitativa de segundo orden en la disciplina psicológica. Se fundamenta en los principios paradigmáticos de la complejidad de Edgar Morin y en los planteamientos de la epistemología cualitativa del psicólogo cubano Fernando González Rey. La investigación cualitativa de segundo orden, en definitiva, reivindica una forma de relación investigador-informante, en las que las experiencias de vida de los informantes y los significados que le asignan se reportan en un clima de horizontalidad, donde prevalece el respeto mutuo, el diálogo reflexivo y se acepta el involucramiento de la subjetividad del investigador en dicho proceso, enmarcado en procesos comprensivos del mismo contexto de desarrollo.

Palabras clave: complejidad, investigación cualitativa de segundo orden, subjetividad.

\footnotetext{
* Artículo de reflexión. Este trabajo surgió como resultado de la participación como estudiante en el espacio académico de Profundización Investigación Cualitativa, liderado por el profesor Juan José Cuervo Rodríguez. 


\title{
Second-order qualitative research and understanding of reality
}

\author{
Abstract \\ This article summarizes some theoretical and personal re- \\ flections about the importance of second-order qualitative \\ research in the psychological discipline. It is based on Edgar \\ Morin's paradigmatic principles of complexity and approa- \\ ches on qualitative epistemology of Cuban psychologist Fer- \\ nando González Rey. Second-order qualitative research, ulti- \\ mately, claims a researcher-informant type of relationship, in \\ which the informant's life experience and the meaning assig- \\ ned to them are reported in a horizontal environment, where \\ mutual respect prevails, reflexive dialogue and in which the \\ involvement of the researchers' subjectivity is accepted in said \\ process, within understanding processes of the same context \\ of development
}

Keywords: complexity, second-order qualitative research, subjectivity. 


\section{Presentación}

¿Qué es lo real? ¿Qué defines cómo real? Si hablas sobre lo que puedes sentir, degustar, oler $y$ ver, entonces lo real simplemente son señales eléctricas interpretadas por tu cerebro.

Matrix

El objetivo de cualquier ciencia es adquirir conocimientos. La elección del método adecuado que nos permita conocer la realidad es, por tanto, fundamental (Cáceres, 1996), pues aún se sigue pensando en que la aspiración metodológica está orientada a descubrir procedimientos y estrategias de investigación que garanticen el estudio de variables donde se generen datos "sólidos y generalizables". De ahí que cuando se habla de la investigación cuantitativa en psicología nos refiramos a entidades con una significación totalmente numérica y descriptiva, que como afirma Hayes (citado en Rodríguez, Gil y García, 1999), produce con frecuencia intentos extremadamente estrictos para medir las conductas humanas o los procesos cognitivos, aun cuando sigue siendo un método totalmente válido para conocer, descubrir y explicar la realidad.

Pero cuando hacemos un "pare", nos introducimos en lo que nos habla la investigación cualitativa en la actualidad -específicamente en psicología-, la cual responde más a una lógica de la construcción humana del conocimiento que a una definición instrumental apoyada en procesos diferentes de construcción de conocimiento, orientada al reconocimiento de la subjetividad desde la articulación de la historia y del contexto de desarrollo. Estas características enmarcan el desarrollo del sujeto y su particularidad, pues no se aíslan las características humanas psicológicas del contexto en que las expresa, sino que, por el contrario, se reconoce al ser humano desde una lógica interactiva o relacional. Aquí se habla de otra forma válida de conocer la realidad, una validez dada en el mismo contexto ecológico.

Pero el punto de vista de este texto no es mirar si existe una especie de antagonismo o complementariedad respecto a estas dos formas de concebir la investigación (lo cualitativo y lo cuantitativo), sino que pretende ofrecer una mirada de cómo la investigación cualitativa de segundo orden emerge como una alternativa que nos posibilita comprender, más que explicar, la realidad. Pero, ¿de qué forma se nos permite acceder a ella? La respuesta la podemos soportar en la afirmación que hace Maturana (1987), que resalta que debido al quiebre de los paradigmas empiristas, estamos actualmente atestiguando la convergencia interdisciplinaria desde la cual se está abriendo un espacio hacia una perspectiva completamente diferente: la de las ciencias de la complejidad.

\section{Filosofía y REALIDAD: BASES EPISTEMOLÓGICAS}

En el Diccionario de filosofía de Ferrater Mora (1964) se define la realidad y lo real como la equivalencia entre ser y existir; distinción que, de una u otra forma, se puede volver verdad, en el sentido de ser siempre y poder accederse a través de los sentidos. La filosofía define la realidad como el conjunto de las cosas existentes, como también las relaciones que estas mantienen entre sí (García, 2005); incluso, los antiguos griegos, generalmente conocidos como los fundadores del pensamiento filosófico occidental (incluyendo a pensadores como Platón, Aristóteles, Parménides, entre otros...), creían que para entender la naturaleza última de 
la realidad primero tenía que entenderse la naturaleza de todas las cosas que hacen parte del extenso universo físico (García, 2005).

Posteriormente, en el siglo XVIII Kant apareció en escena, como plantea Alpher (2008) en el análisis del libro Crítica de la razón pura. En este libro Kant dio uno de los pasos más revolucionarios en la historia: conceptualizó el pensamiento humano, sugiriendo que para entender la realidad, el ser humano necesitaba orientar la dirección de las preguntas desde afuera hacia adentro. Kant propuso que no se debía estudiar la naturaleza de los objetos, sino el modo en que estos son percibidos, señalando la necesidad de mirar hacia el interior, hacia la naturaleza de la percepción misma, llegando con esto a refutar a John Locke, para quien la mente era una tábula rasa.

Para Kant, el ser humano tiene modelos de percepción incorporados tanto de orden espacial como temporal, que nos permiten organizar la información que los órganos sensoriales nos trasmiten constantemente. Esta idea, que Jean Piaget logró demostrar con sus estudios, señala que existen modos innatos de comprensión que orientan la forma en que los seres humanos entiende e interpretan la realidad, lo cual se evidencia en la etapa de "la fase de las operaciones concretas", sugiriendo así que las actitudes témporo-espaciales representan una parte intrínseca del desarrollo cognitivo del ser humano.

En contraste, dentro de las posturas filosóficas existen las llamadas "teorías realistas u ontológicas", las cuales dan una valoración superior al objeto, de tal modo que este se impone, y el sujeto recibe su impacto. La función del sujeto, entonces, es de pasividad. Desde estas teorías existe una postura realista expuesta por Mario Bunge, que distingue tres matices de realidad: el llamado "realismo ingenuo", que sostiene que existe un mundo real y que es sustancialmente tal como lo percibimos. Este realismo sostiene que el mundo real coincide con el mundo percibido y que es independiente del sujeto. Otro matiz concibe el llamado "realismo crítico", que considera que hay que distinguir entre el mundo físico y la percepción que hacemos de él, pero que en definitiva es el objeto el que se impone al sujeto. Por último se encuentra el "realismo científico", que sostiene que el conocimiento sobre fenómenos independientes de la teoría es el producto de una investigación científica exitosa, y que tal conocimiento se hace posible aun en aquellos casos en que el fenómeno relevante no se pueda observar directamente. De acuerdo con el realismo científico, por ejemplo, si la persona adquiere un buen libro de texto contemporáneo sobre química, tendrá buenas razones para creer que son verdaderas las aseveraciones que contiene sobre la existencia y las propiedades de los átomos, moléculas, partículas subatómicas, niveles de energía, mecanismos de reacción, etc. (Boyd, 2002).

Otra propuesta interesante para esta discusión es la que realiza Maturana, quien hace explícita la importancia de dar respuesta a la pregunta sobre ¿qué es la realidad? El autor sostiene que la respuesta explícita o implícita que cada ser humano da a este interrogante determina cómo cada quien vive su vida, cómo se acepta o rechaza a los demás seres humanos en la red social que este integra o no, y cómo el hombre comprende adecuadamente los fenómenos sociales y no sociales. Maturana (1995) resalta que esta pregunta puede ser adecuadamente 
respondida, considerando al observador como una entidad biológica:

El camino de la ciencia moderna no es el camino de la sabiduría de vivir en los mundos en que habitamos los seres humanos, aunque no forzosamente lo tiene que contradecir. El camino de la sabiduría, al igual que el camino de la comprensión, la preocupación y la responsabilidad por las consecuencias de nuestras acciones en los mundos que los seres humanos ponemos de manifiesto y vivimos, deben ser cultivados en especial si es que han de tener alguna presencia en nuestra vida diaria (p. 89).

En síntesis, identificar nuestras formas de acceso a la realidad es fundamental, ya que desde la perspectiva de la comprensión de las posibilidades de conocimiento de la realidad humana, se acepta la existencia de múltiples visiones con grados de entendimiento y validez sobre dicha realidad. En este sentido, es particularmente importante, para las opciones investigativas de tipo cualitativo, reconocer que el conocimiento de la realidad humana supone no solo la descripción operativa de ella, sino ante todo la comprensión de su sentido por parte de quienes la producen y la viven (Sandoval et ál., s.f. ). Además, siguiendo la perspectiva de la complejidad, para ser objetivos es imprescindible reconocer que lo exterior existe sin necesidad y en absoluta independencia de nosotros (Jaillier et ál., s.f.).

Atkinson (citado en Vicente, 2008) plantea que en los últimos veinte años la investigación cualitativa ha ido floreciendo a una escala global, a pesar de que como práctica tiene más de cien años, desde que Glasser y Strauss en 1967 la establecieron como una fuerza metodológica de investigación (Gobo, 2005). Esa fuerza metodológica, como señalan Taylor y Bogdan (1992), está dada por la manera como enfocamos los problemas y buscamos las respuestas a estos. Al respecto, Blúmer (1969, citado en Bahamón, 2008) plantea:

Las personas actúan con respecto a las
cosas e inclusive frente a las personas
sobre la base de los significados que
unas y otras tienen para ellas; los sig-
nificados son productos sociales que
surgen durante la interacción; los ac-
tores sociales asignan significados a si-
tuaciones, a otras personas, a las cosas
y a sí mismos a través de un proceso de
interpretación (p. 137).

Dicho de otro modo, toda realidad humana se concibe como una realidad desarrollada simultáneamente sobre tres planos: físicomaterial, sociocultural y personal-vivencial, cada uno de los cuales posee lógicas particulares de acceso para su comprensión. Así, se debe dar paso a la reflexión, para abordar los órdenes de lo ético, lo político, lo cultural, que es muy significativo en los planos sociocultural y personal-vivencial (Sandoval et ál., 1996). Es en estos dos últimos planos en donde se construyen lo subjetivo y lo intersubjetivo, en tanto objetos y vehículos de conocimiento de lo humano (Hoyos y Vargas, 1997).

\section{SubJeTIVIDAD: OBJETO DE ESTUDIO DE LA INVESTIGACIÓN CUALITATIVA DE SEGUNDO ORDEN}

Sandoval et ál. (1996) resaltan que dentro de la investigación cualitativa se intenta: a) recuperar la subjetividad como espacio de construcción de la vida humana; b) reivindicar la vida cotidiana como escenario básico para comprender la realidad sociocultural; y c) comprender la intersubjetividad y 
el consenso como vehículos para acceder al conocimiento válido de la realidad humana.

La investigación cualitativa ha expandido su dominio debido a que los procesos de transformación de nuestras sociedades contemporáneas se están concretando en procesos más simbólicos y subjetivos, en tanto son modos de construir identidad y de significar los espacios y procesos sociales (Flick, 2002). Este último autor resalta que la investigación cualitativa se ha defendido como una práctica que intenta rescatar al sujeto de su cualificación como objeto por parte de las metodologías positivistas. Este rescate de la condición de sujeto implica la constitución de la práctica de investigación como un ejercicio que reconoce en el otro un saber a ser explorado, es decir, que el investigador es capaz de abrir al diálogo, dejándose enseñar de los otros. Allí emergerá una verdad que está dada por una participación conjunta y contextualizada; en conclusión, tanto el investigador como los participantes se pueden considerar entes activos del proceso a investigar.

González (2008) hace hincapié en que desde una perspectiva histórico-cultural, la subjetividad abre nuevas opciones para el desarrollo de las representaciones sociales $y$, sobre todo, permite una integración entre lo individual y lo social; a su vez resalta que lo subjetivo aparece mucho más como una referencia genérica para significar procesos del sujeto que conoce y construye, y no tanto como una definición ontológica particular de los fenómenos humanos. Análogamente resalta que la separación de lo individual y lo social no permite ver que la organización psíquica individual se desarrolla en la experiencia social e histórica de los individuos, y tampoco permite considerar cómo las acciones de los individuos -que son inseparables de su producción subjetiva- tienen un impacto que, de hecho, se asocia a nuevos procesos de transformación de las formas de vida y organización social (González et ál., 2008). He aquí la importancia de resaltar el pensamiento histórico-cultural de Vygotsky. Fue allí donde Fernando González Rey desarrolló sus trabajos sobre la subjetividad como objeto de estudio de la investigación cualitativa de segundo orden en psicología.

González (2008) considera que el sentido subjetivo expresa las producciones simbólicas y emocionales, configuradas en las dimensiones histórica y social de las actividades humanas; es decir, estas no expresan solo el momento actual de un sistema de relaciones, sino la historia tanto de las personas implicadas en un espacio social como de ese espacio social en su articulación con otros; por ende, según este autor, la subjetividad no es exclusiva de experiencias individuales, sino que existe una relación directa con los diferentes espacios de vida social de los individuos. La llamada "subjetividad social" (González et ál., 2008) es aquella forma que logra integrar todos los sentidos subjetivos dados por una interrelación entre los diferentes espacios sociales (familia, trabajo, colegio, banda de amigos, etc.), que está alimentando constantemente la subjetividad individual dada en espacios sociales. En conclusión, González et ál. (2008) señalan:

La persona es la portadora de procesos subjetivos en su tránsito simultáneo por múltiples espacios sociales. La persona es un sistema complejo en los múltiples sistemas sociales en que actúa (p. 235). 
Gómez y González (2005) consideran que retomar la perspectiva histórico-cultural dentro de la investigación cualitativa de segundo orden (epistemología cualitativa) implica la posibilidad de construir una subjetividad en la que se rechaza cualquier forma de determinismos, de leyes, pero en la que se pueden rescatar todas aquellas valoraciones culturales; pero también está el ámbito cultural en el que el investigador genera sentidos sobre la subjetividad que se ha investigado. La psicología histórico-cultural, desde sus orígenes (Vigotsky, 1989), ofrece una concepción del ser humano y de la naturaleza, los cuales ser articulan en niveles sociales y psicológicos dentro de un marco dialéctico; se trata, fundamentalmente, de un intento por superar la dicotomía entre lo social y lo individual.

Desde el enfoque histórico-cultural, la subjetividad ofrece un campo de investigación fundamentado en la complejidad (Morin, 1998) y en la epistemología cualitativa (González, 1997), cuyos elementos esenciales giran en torno a la construcción del sentido, al sujeto como generador y constructor de ellos y a la inclusión de su dimensión afectiva dentro de la configuración subjetiva. La subjetividad no se reduce a un estado interno, sino a una dimensión compleja que involucra tanto lo psicológico como lo social, en una relación dialéctica y cuya naturaleza es histórica y social (Hernández et ál., 2008). Por esta razón, se propone una manera de tránsito desde el pensamiento dialéctico hacia el pensamiento complejo. La subjetividad debe entenderse no como una dimensión dialéctica, sino como una dimensión compleja (Hernández et ál., 2008).

Particularmente, de la subjetividad subyacen aquellos principios derivados y que se encuentran latentes en su propia construcción teórica y conceptual; ellos son el principio dialógico, el principio de recursividad organizacional y el principio hologramático. Esa derivación puede entenderse como el resultado de enmarcar y/o situar la categoría conceptual de la subjetividad humana dentro del pensamiento complejo. Adicionalmente, los principios señalados permiten un acercamiento a la subjetividad desde el enfoque histórico cultural (Hernández et ál., 2008).

Continuando con lo anterior, desde la perspectiva histórico-cultural se trata de comprender que la subjetividad no es algo que aparece solo en el plano individual, sino que la propia cultura en la cual se constituye el sujeto individual (y de la cual es también constituyente) representa un sistema subjetivo generador de subjetividad (González, 2002). Así, entonces, yo no construyo mi subjetividad, sino que mi subjetividad se constituye en un campo de mi acción. Por eso se habla de la construcción de la subjetividad desde las historias de vida, en las que emergen muchos protagonistas dentro de un conjunto de campos. De ahí viene la perspectiva de la complejidad, de la multiplicidad de factores que están presentes en su constitución (Gómez et ál., 2005).

Se puede decir, entonces, que dentro de la investigación cualitativa se ha logrado reivindicar una forma de relación investigador-informante, en la que las experiencias de vida de los informantes y los significados que les asignan se reportan en un clima de horizontalidad, donde prevalece el respeto mutuo, el diálogo reflexivo y se acepta el involucramiento de la subjetividad del investigador en dicho proceso (Wisenfield, 2000). La pregunta que de aquí surge quizás 
es: ¿cómo se garantiza la neutralidad del investigador? Vicente (2008) responde: “La neutralidad del investigador se garantiza por su experticia y adecuado uso del instrumental" (p. 119)

La noción de recolectar datos lo muestra: los datos están ahí, en este caso los significados; a partir del uso correcto de nuestras herramientas podremos recolectarlos y clasificarlos en las categorías que nuestro saber experto indica. Sin embargo, el giro lingüístico, el posestructuralismo, el desarrollo del construccionismo social, entre otros, coinciden en señalar que los significados que pueden ser "recolectados" emergen de prácticas sociales situadas, y es ahí donde son construidos y reconstruidos (Vicente et ál., 2008).

Finalmente, la noción de la subjetividad desde el enfoque histórico-cultural rompe con un conjunto de dicotomías presentes en la tradición de la psicología y comienza a ubicarse dentro del pensamiento complejo, en sus implicaciones metodológicas para la investigación y en las exigencias de un rol del investigador diferente al tradicionalmente aceptado (Hernández et ál., 2008 ).

\section{LA REALIDAD COMO PROCESO DE SIMBOLIZACIÓN-SUBJETIVACIÓN}

Es así que la investigación cualitativa ha expandido su dominio, debido a los procesos de transformación de nuestra sociedad contemporánea, concretando procesos simbólicos y subjetivos, lo que da paso a construir identidad y significar los espacios y los procesos sociales (Flick, 2002).

Hoy por hoy, la definición sobre la investigación cualitativa va más allá de la definición que nos dan Hernández, Fernández y
Baptista (2006) en su libro Metodología de la investigación: “La Investigación cualitativa es un paso exploratorio de proceso inductivo en el que los objetivos y las preguntas de investigación son más generales y su delimitación es menos precisa" (p. 527). Pero es importante saber que las preguntas de la investigación cualitativa van más allá de considerar: ¿cómo se interpreta la realidad?, ¿cómo resignificamos la realidad?, ¿cómo el ser humano integra la realidad? (Palabras como "interpretar", "resignificar" e "integrar" se han convertido en clichés, pues en la investigación cualitativa la cantidad de conceptos que se pueden utilizar son más amplios y ricos; he aquí algunos ejemplos: capturar, explorar, elaborar, sistematizar, conectar, reivindicar, representar, co-construir, reconstruir, representar, trasformar, crear...). Añado a esto que los investigadores cualitativos son sujetos activos e interactuantes con la realidad que están estudiando; son observadores especializados con herramientas teórico-metodológicas especializadas, que les permitirán ver cómo los otros construyen su mundo e, incluso, cómo él mismo participa en la construcción de su mismo mundo. En este sentido, como resalta Sandoval (1996), es particularmente importante, para las opciones investigativas de tipo cualitativo, reconocer que el conocimiento de la realidad humana supone no solo la descripción operativa de ella, sino ante todo la comprensión del sentido de esta por parte de quienes la producen y la viven.

En este punto es importante resaltar que la investigación cualitativa de segundo orden es epistemológicamente reflexiva y contextual. No es, como lo resaltaba en el párrafo anterior, una mera descripción operativa, sino una acción en la que se incluye al sujeto en la observación de sus estudios científicos y en 
la que este es capaz de hacer conciencia de que lo que está observando también lo construyen otros observadores (Mejía, 2002).

Una disciplina como la psicología, que se reconfigura en la construcción teórica y que quiere sustentarse a través de fundamentos investigativos, debe consolidarse a través de posturas filosóficas, epistemológicas $\mathrm{y}$ paradigmáticas que permitan lograr tales objetivos, y es en la investigación cualitativa de segundo orden, como manera de hacer viva la complejidad, donde los elementos esenciales giran en torno a la construcción de ese sentido, el sujeto como generador y constructor de ellos y a la inclusión de su dimensión afectiva dentro de la configuración subjetiva (González, 2000). Así, la subjetividad no se avasalla, sino que, por el contrario, es tomada como una dimensión psicológica en la que se involucra tanto la subjetividad social como la subjetividad individual. Análogamente, estas son las contribuciones que ha hecho la psicología soviética respecto a la subjetividad como objeto de estudio de la psicología. Autores como Vigotsky y Rubinstein lograron integrar lo social y lo cultural, conformándolos como un todo en el que están interrelacionados (Hernández, 2008). De aquí surgen los fundamentos epistemológicos y ontológicos sobre la subjetividad:

Tenemos que reemplazar la visión mecanicista de ver la cultura, sujeto y subjetividad como fenómenos diferentes que se relacionan, para pasar a verlos como fenómenos que, sin ser idénticos, se integran como momentos cualitativos de la ecología humana en una relación de recursividad (González, 2002, p. 164).

Actualmente, el concepto de subjetividad ha sido ligado a la concepción del pensamiento complejo de Morin. González (2002) sustenta que la subjetividad es un sistema dialéctico y complejo en donde el sujeto responde a la comprensión del conjunto de tensiones, contradicciones e interrelaciones. Todo esto converge en superar los pensamientos reduccionistas y fragmentados de corrientes positivistas como el conductismo o el cognitivismo conductual, los cuales no ofrecen un acercamiento hacia la diversidad de la psique humana, como se había resaltado anteriormente.

Por eso, como nos lo presenta Jaillier (2003), el interés de estudiar la realidad surge desde la investigación cualitativa de segundo orden a partir de preguntas como: ¿es la realidad, entonces, una construcción relacional, relativa y múltiple que está a la vez fuera y dentro de nuestro espíritu? De aquí surge también el interés del presente texto por responder a las preguntas: ¿cómo la investigación cualitativa de segundo orden nos permite comprender la realidad?, ¿de qué forma se nos permite acceder a ella? La investigación social de segundo orden, como lo llama Jaillier (2003), contribuye a la búsqueda de una verdad que no es una, sino que acepta las múltiples realidades contrapuestas, al hacerse consciente de los modelos o paradigmas de ciencia impuestos que llevan a un riesgo permanente de ilusión (utopía). Y como lo resalta también Morin (1994), la investigación social de segundo orden tiene como objeto ser al mismo tiempo investigador-investigado, sujeto-objeto, por lo que el punto de vista cambia de posición y se convierte en un punto de vista metasociológico, metahistórico y metaantropológico que permite la reflexividad.

La investigación cualitativa de segundo orden definitivamente permite darnos un 
abordaje de la realidad, a partir de lo cual podemos hallar que las personas, los contextos o los grupos no son reducidos, sino que son considerados como un todo, es decir, cuando se es capaz de estudiar a las personas en el contexto de su pasado y las limitaciones en las que se halla; cuando como investigadores somos capaces de ir más allá de la entrevista estructurada y somos capaces de dialogar y conversar con el otro; cuando somos capaces de identificarnos con el otro para comprender cómo experimenta su realidad, dando paso a procesos autorreferenciales e intersubjetivos; cuando como investigadores somos capaces de no comparar, sino de ver la realidad como si fuese la primera vez que sucediese; cuando el investigador es capaz de comprender detalladamente las perspectivas de las personas sin la necesidad de buscar la verdad o la moral de eso que el otro nos está compartiendo; cuando como investigadores dejamos de generalizar y nos damos cuenta de que cada ser humano en el mundo es único, complejo e irrepetible; finalmente, cuando el investigador es capaz de ver que la realidad es única del sujeto y no es capaz de subrayarla solamente como "datos" válidos y confiables. Como resalta Erickson (1982), "la investigación cualitativa pretende acceder al significado de las acciones desde la perspectiva del actor". Apoyando esto, Fromm (1983) ejemplifica:

La psicología del comportamiento puede ser una ciencia, pero no es ninguna ciencia del hombre alienado con métodos alienados, realizada por investigadores alienados. Está por cierto en condiciones de poner en relieve ciertos aspectos del hombre, pero justamente lo vivo, lo específicamente humano, ni lo roza (p. 28).
Por ejemplo, Mertens (2005) resalta que no hay una realidad objetiva; la realidad es edificada socialmente. En consecuencia, existen múltiples construcciones mentales que pueden ser "aprehendidas", algunas de las cuales pueden estar en conflictos con otras. De este modo, las concepciones de la realidad son modificadas a través del tiempo. En definitiva, la investigación cualitativa de segundo orden reivindica una forma de relación investigador-informante, en las que las experiencias de vida de los informantes y los significados que le asignan se reportan en un clima de horizontalidad, donde prevalece el respeto mutuo, el dialogó reflexivo y se acepta el involucramiento de la subjetividad del investigador en dicho proceso (Wiesenfeld, 2000).

He aquí la importancia de hablar de las dos dimensiones de la subjetividad de las que habla González (2005): la subjetividad social y la subjtetividad individual, que como él resalta, no son divisiones dicotómicas, sino que expresan dos momentos de unidad. Por ejemplo, la subjetividad social cumple con una característica esencial: se desarrolla en la sociedad en donde se crean significados y sentidos, pues es en la vida donde se engendra culturalmente y en donde se constituye su historia. Y la subjetividad individual cumple con características como que es propia del sujeto, versátil, flexible, compleja, plurideterminada; aunque es determinada socialmente, no quiere decir que sea lineal, pues está en constante proceso de constitución. Tanto la subjetividad social como la individual pueden verse afectadas debido a las condiciones del sujeto.

Así, la subjetividad debe tomarse como objeto de estudio de la investigación cualitativa de segundo orden, pues todo esto 
constituye su gran complejidad. También es una investigación que permite conceptualizar el objeto de estudio de la psicología -la psique, la mente, la conciencia- e impedir la clasificación de esta como solamente una función cerebral, medible, manipulable y/o controlable. Por ende, cuando se asuma el sello característico del investigador cualitativo, se debe tener una disposición de apertura y aprendizaje permanentes, ejercidos mediante un esfuerzo cotidiano de escucha, observación, reflexión y diálogo sistemáticos y respetuosos; asimismo, se estará en la posibilidad de visualizar y comprender las intimidades y profundidades del amplio y diverso multiverso ${ }^{1}$ de la realidad humana.

La investigación cualitativa de segundo orden, entonces, busca esas realidades relativas y reflexivas, no aprisiona el conocimiento en el aquí y el ahora, permitiendo que el acto mismo de conocer no se inscriba en un punto de vista particular o fijo, sino que aun si existe ese punto de vista, se pueda buscar un metapunto de vista que supere al inicial, y de este modo se conceda la posibilidad de reflexionar y relativizar los conocimientos (Jaillier, 2003).

Morin (1998) nos permite ver que dentro de la recolección de información del investigador cualitativo, el traslado de la información recogida por él a la población con la que se estuvo trabajando se transforma en un proceso considerado como "transducción", y gracias a este logra una comprensión

"Multiverso" es un término usado para definir los múltiples universos posibles, incluido nuestro propio universo. Comprende todo lo que existe físicamente: la totalidad del espacio y del tiempo, todas las formas de materia, energía y cantidad de movimiento, y las leyes físicas y constantes que las gobiernan. El concepto de multiverso ha sido supuesto en cosmología, física, astronomía, filosofía, psicología transpersonal y ficción, en particular dentro de la ciencia ficción y de la fantasía. El término fue acuñado en 1895 por el psicólogo William James. de mayor complejidad de la realidad. De aquí parte la importancia de hablar de lo dialógico:

La dialógica de los puntos de vista es uno de los constituyentes del conocimiento del conocimiento que, al permitir la entre-articulación de sus diversas instancias constitutivas, permite al mismo tiempo los metapuntos de vista (Gómez, 2008, p. 3).

Articulando esto con la investigación cualitativa de segundo orden, su centro es el aquí y el ahora -el contexto-, donde se plantea que lo real va más allá de lo pensable, pero que existe. Esta visión, como resaltan Jaillier et ál. (2003), permite dar herramientas en el manejo del mundo que no es a través de la simplificación, sino de hacer de este algo más rico: el conocer del conocer.

El ser humano siempre ha intentado estar inmerso en la realidad, acercándose quizás a la verdad de las cosas, ampliando su conocimiento; y para eso ha sido necesario disminuir la ficción, la fantasía y la ilusión, pues como codiciosos expresan una gran necesidad esencialmente humana: la necesidad de relacionarse y afirmarse en el mundo como un "alguien"; y es en realidad donde se pueden relacionar de manera humana con lo que los rodea; incluso toda relación con la realidad le permitirá ensancharse con el conocimiento, y la historia del ser humano es una historia de crecimiento cognoscente.

Hoy por hoy se espera que los investigadores poco a poco transformen su pensamiento reduccionista y simplificador de la realidad y opten por un pensamiento descentralizado, no generalizado per se, pues hasta el mismo Einstein nos demostró, gracias a su teoría de la relatividad, que ni el espacio ni el tiempo son absolutos. También 
lo demuestran la teoría de supercuerdas de Joel Scherk y John Schwuarz y la teoría M, donde se resalta la formación de múltiples dimensiones y consideran plausible la existencia de más universos. Todo esto está fundamentado en teorías como la cuántica y la de Kulaza-Klein (Martínez, 2006).

El "psicologizar" acerca de la realidad es de escaso valor si nos mantenemos lejos del observador; es una idea que se convertiría en abstracta y llena de retórica, en lugar de ponerla como el objetivo esencial del estudio, de referirla al ser humano y a sus experiencias. La auténtica psicología existe solamente compartiendo las profundas experiencias humanas, no en el encasillamiento.

Las ideas y las investigaciones dentro de nuestra disciplina llegarían a ser poderosas únicamente si se adquiere el valor del ser humano y si la investigación en psicología va más allá, como lo ha indicado González Rey. Entonces ha de tener influencia y es menester sembrarla en tierra fértil: investigaciones con un valor potencial más profundo y más enriquecedor. A fin de "analizar y experimentar" el mundo, se trataría de comprender mejor el mundo, de trascender y ser más abiertos, de forjar sus prejuicios y tomar riesgos. Así se superaría y desarrollaría con disciplina la osadía de un arraigo profundo en sí mismos y la convicción y la plena relación con la humanidad y lo que esta nos puede dar. Allí la psicología no ha de ser vista como una disciplina individualista.

En conclusión, el mundo está cambiando rápidamente. Estamos enfrentando a lo que Erich Fromm llamó en su libro La Revolución de la esperanza como homo tecnologicus, y para esto la psicología debe enfrentar los problemas que esta sociedad del siglo XXI nos está acarreando. Hay que conocer y comprender el mundo, y para eso debemos cambiar la forma de investigar la realidad. $Y$ todo debe darse desde la academia, debe pensarse desde ya en que debemos cambiar la forma de observar al ser humano, entonces podremos hacer parte reflexiva de estas nuevas dinámicas sociales e intentar reflexionar de otra forma las problemáticas cotidianas.

\section{Referencias}

Alpher, M. (2008). Dios está en el cerebro: una interpretación científica de la espiritualidad humana y de Dios. Bogotá: Norma

Bahamón, P. (2008). Configuración de la ética ciudadana en el corrido prohibido análisis semiótico. Recuperado de http://revistas.uis.edu.co/index.php/ revistaS/article/view/228/1062

Boyd, R. (2002). Realismo científico. Asociación Oaxaqueña de Psicología. Recuperado de http://www.conductitlan.net/ seminarios/realismo_cientifico.pdf

Cáceres, A. (1996). El método científico en las ciencias de la salud. Las bases de la investigación biomédica. Madrid: Díaz de Santos.

Erickson, F. (1982). La investigación en la enseñanza. Barcelona: Paidós.

Ferrater, J. (1964). Diccionario de filosofía (tomo II). Buenos Aires: Suramericana

Flick, U. (2002). Introducción a la investigación cualitativa. Madrid: Morata.

Fromm, E. (1983). El amor a la vida. Buenos Aires: Paidós.

García, F. (s.f.). La filosofía como racionalidad teórica: conocimiento, verdad, realidad. Recuperado de http://www.aafi. filosofia.net/didactica/apuntes/primero/tema3.pdf 
García, M. (2005). Lecciones preliminares de filosofía. Bogotá: Ediciones Universales.

Gobo, G. (2005). The renaissance of qualitative methods. Recuperado de http://nbn-resolving.de/urn:nbn:de: 0114-fqs0503420

Gómez, H. (2008). Cismogénesis y morfogénesis del conocimiento. Los estudios de la comunicación y la sociología cultural. Recuperado de http://redalyc.uaemex. $\mathrm{mx} /$ src/inicio/ForazarDescargaArchivo. jsp?cvRev=1995\&cvArt=199520720006 \&nombre=Cismog $\%$ E9nesis $\% 20 y \% 20$ morfog\%E9nesis\%20del\%20conocimiento.\%20Los\%20estudios\%20de\%20 la\%20Comunicaci\%F3n \%20y\%20la $\% 20$ Sociolog\%EDa\%20Cultural

Gómez, A. y González, F. (2005). Subjetividad: una perspectiva histórico-cultural. Conversación con el psicólogo cubano Fernando González Rey. Recuperado de http://redalyc.uaemex.mx/src/inicio/ ArtPdfRed.jsp?iCve=64740311

González, F. (1997). Epistemología cualitativa y subjetividad. La Habana: Pueblo y Educación.

González, F. (2000). Investigación cualitativa en psicología. Rumbos y desafíos. México: Thomson.

González, F. (2002). Sujeto y subjetividad, una aproximación histórico-cultural. Buenos Aires: Thomson.

González, F. (2005). El sujeto y la subjetividad: algunos de los dilemas actuales de su estudio. Brasilia: Universidad de Brasilia.

González, F. (2008). Subjetividad social, sujeto y representaciones sociales. Recuperado de http://www.usta.edu.co/ otras_pag/revistas/diversitas/doc_pdf/ diversitas_8/vol.4no.2/articulo_1.pdf
Hernández, O. (2008). La subjetividad desde la perspectiva sociocultural: un tránsito desde el pensamiento dialectico al pensamiento complejo. Revista Colombiana de Psicología, 17, 147-160.

Hernández, Fernández y Baptista (2006). Metodología de la investigación. México: McGraw-Hill.

Hoyos, G. y Vargas, G. (1997). La teoría de la acción comunicativa como nuevo paradigma de las ciencias sociales: las ciencias de la discusión. Recuperado de http:// es.scribd.com/doc/81239652/La-Teoriade-La-Accion-Comunicativa-ComoNuevo-Paradigma

Jaillier, E. (2003). Realidad y visión investigativa social-reflexiva del mundo: entre la utopía y lo real más allá de lo pensable. Red de Revistas Científicas de América Latina, el Caribe, España y Portugal, 18, 64-70.

Martínez, E. (2006, 29 de enero). Científicos norteamericanos detectan la existencia de dimensiones adicionales: las colisiones de neutrinos de alta energía con otras partículas corroboran uno de los postulados de la Teoría de Supercuerdas. Tendencias Científicas. Recuperado de http://www.tendencias21.net/ Cientificos-norteamericanos-detectanla existencia-de-dimensiones-adicionales_a861.html

Maturana, H. (1987). Biology of language: the epistemology of reality. Recuperado de http://www.enolagaia.com/ M78BoL.html

Maturana, H. (1995). La realidad: ¿objetiva o construida? Fundamentos biológicos de la realidad. México: Antrhopos.

Mejía, J. (2002). Perspectiva de la investigación social de segundo orden. 
Recuperado de http://redalyc.uaemex. mx/pdf/101/10101405.pdf

Mertens, D. (2005). Research and evaluation in education and psychology: integrating diversity with quantitative, qualitative and mixed methods. United States of America: SAGE.

Morin, É. (1994). El método III. El conocimiento del conocimiento. Madrid: Cátedra.

Morin, É. (1998a). El método IV. Las ideas. Madrid: Cátedra.

Morin, É. (1998b). Introducción al pensamiento complejo. Barcelona: Gedisa.

Rodríguez, G., Gil, J. y García, E. (1999): Metodología de la investigación cualitativa. Granada: ALJIBE.

Sandoval, A. (1996). Programa de Especialización en Teoría, Métodos y Técnicas en Investigación Social: Investigación
Cualitativa. Recuperado de http://sapiens.ya.com/metcualum/sandoval.pdf

Taylor, S. J. y Bogdan, R. (1992) Introducción a los métodos cualitativos de investigación. Barcelona: Paidós.

Vicente, S. (2008). La investigación como una aventura de producción dialógica: la relación con el otro y los criterios de validación en la metodología cualitativa contemporánea. Psicoperspectivas, 7 , 114-136.

Vigotsky, L. (1998). La formación de las funciones psíquicas superiores. Obras escogidas. Buenos Aires: Paidós.

Wiesenfeld, E. (2000). Entre la prescripción y la acción: la brecha entre la teoría y la práctica en las investigaciones cualitativas. Recuperado de http://qualitativeresearch.net/fqs/fqs-e/2-00inhalt-e.html 